\title{
Anti-Inflammatory and Anti-Oxidant Activity of Ultra-Short Wave Diathermy on LPS-Induced Rat Lung Injury Q. Wu ${ }^{1,2}$, M. Qu ${ }^{2}$, P. Zhong' ${ }^{2}$ Y. Zeng' ${ }^{2}$ J. Wang ${ }^{2}$, Q. Zhang ${ }^{2}$, Ting Wang', D. Liu' ${ }^{2}$ L. Yang', J. Zhou ${ }^{2}$, and Tong Wang ${ }^{1}$
}

Translated from Byulleten'Eksperimental'noi Biologii i Meditsiny, Vol. 172, No. 10, pp. 432-439, October, 2021 Original article submitted April 7, 2021

\begin{abstract}
We studied the lung-protective effect and mechanisms of the anti-inflammatory and antioxidant effects of ultra-short-wave diathermy (USWD) in a rat model of LPS-induced acute lung injury. Histological examination of the lung tissues was performed and the levels of oxidative stress-related factors and inflammatory cytokines were measured. It was shown that the lung injury score, the lung wet-to-dry weight ratio (W/D), oxidative stress-related factors malondialdehyde and acyl-CoA synthetase long-chain family member 4 (ACSL4), and inflammatory cytokines were increased after LPS administration, while USWD treatment reduced these parameters. In addition, superoxide dismutase and glutathione peroxidase 4 were decreased in rats with LPS-induced acute lung injury, while USWD therapy up-regulated the expression of these enzymes. Thus, USWD could antagonize lung injury by inhibiting oxidative stress and inflammatory response in rats with acute lung injury. USWD can be a promising adjunctive treatment to counter oxidative stress and inflammation and a potential therapeutic candidate for the treatment of patients with this pathology.
\end{abstract}

Key Words: ultra-short wave diathermy therapy; acute lung injury; oxidative stress; inflammation

Acute lung injury (ALI) leads to acute respiratory distress syndrome (ARDS) characterized by high morbidity and mortality. The critical pathological characteristics of ALI include accumulation of neutrophils, interstitial edema, and damage to the alveolar epithelium of the lungs [9]. Despite great efforts to find new and/or more effective treatments for ALI and ARDS and discovery of pathological factors, mortality remains high [8]. Ultra-short wave diathermy therapy (USWD) is one of the oldest forms of electrotherapeutic modality traditionally used by physical therapists to treat various inflammatory diseases. Many studies have demonstrated that USWD is capable of relieving the inflammation and has been suggested as promising adjuvant to drug-based therapies [15]. The beneficial

${ }^{1}$ The First Affiliated Hospital with Nanjing Medical University, Nanjing, Jiangsu, China; ${ }^{2}$ The First Affiliated Hospital of University of South China, Department of Rehabilitation, Hengyang Medical School, University of South China, Hengyang, Hunan, China. Address for correspondence: wangtong60621@163.com. Tong Wang effects of USWD in ALI patients were also reported, however, the mechanisms underlying these effects are still largely unknown.

The physiological effects attributed to USWD include increased regional blood flow, decreased soft tissue pain and stiffness, reduced inflammation, relieved edema, and healed wounds, which are probably a result of the ability of cells to absorb energy from oscillating electrical fields of defined frequencies and/ or amplitudes [11]. Due to anti-inflammatory property of USWD, its application for the treatment of ALI patients has increased dramatically in the last 20 years [15]. The pathophysiological process of ALI is recognized as an excessive, uncontrolled inflammatory response in the lungs. Oxidative stress followed by hyperinflammation described as the "cytokine storm" can cause the ALI and has been regarded as the trigger of lung injury [3]. Many clinical studies have verified the protective effects of USWD on the lungs in ALI patients (Zhang, et al., 2003). However, whether these 
effects are related to the regulation of inflammation and oxidative stress in the lungs remains to be investigated.

This study aimed to ascertain whether USWD could antagonize the oxidative stress and inflammatory response in rats with LPS-induced ALI. To this end, oxidative stress-related factors superoxide dismutase (SOD) and malondialdehyde (MDA), LPO-related factors acyl-CoA synthetase long-chain family member 4 (ACSL4) and glutathione peroxidase 4 (GPx4), inflammatory cytokines were measured and lung histopathology was studied.

\section{MATERIALS AND METHODS}

Animal model and experimental design. Male Sprague-Dawley rats $(n=18$, age 3 months, weight 333-505 g) were purchased from Hunan Slack Jingda Experimental Animal Co., Ltd. The animals were randomly divided into three groups (6 rats per group): control, ALI without treatment, and ALI+USWD treatment. The rats were housed in cages under controlled conditions $\left(55 \pm 5 \%\right.$ relative humidity, $24 \pm 2{ }^{\circ} \mathrm{C}$, and a $12 / 12 \mathrm{~h} \mathrm{light/dark}$ cycle) with unrestricted access to water and food for at least 7 days before ALI modeling. ALI was modeled by intratracheal instillation of LPS ( $5 \mathrm{mg} / \mathrm{kg}, 5 \mathrm{mg}$ LPS was dissolved in $5 \mathrm{ml} \mathrm{PBS}$ ). Rats of the control group received an equal amount of PBS via the same route [3] (Chimenti, et al., 2017). In $24 \mathrm{~h}$ after instillation of LPS or PBS (control), the rats were sacrificed under chloral hydrate $(300 \mathrm{mg} / \mathrm{kg})$ anesthesia.

All experimental procedures and protocols were approved by the Ethics Committee of the First Affiliated Hospital of University of South China (Medical Experimental Animal Number: SCXK 2019-0004).

USWD therapy. USWD was performed for $15 \mathrm{~min}$ immediately and 4 and $8 \mathrm{~h}$ after ALI modeling using an ultra-short wave diathermy device (Shanghai Electrical Device Company; frequency $40.68 \mathrm{MHz}$, intensity $50 \mathrm{~mA}$, maximum output power $11.32 \mathrm{~W}$ ). A pair of disc electrodes were placed bilaterally at a distance of $2 \mathrm{~cm}$ from the skin. All rats in LPS and ALI groups received fake USWD exposure.

Histopathology. After sacrifice, the lungs were isolated and immediately divided into lobes. The right upper lobe was weighed on a weighing disk (wet weight, W) and then dried in a constant temperature oven at $60^{\circ} \mathrm{C}$ for $48 \mathrm{~h}$ until constant weight (dry weight, D). The lung wet/dry weight ratio (W/D) was calculated to evaluate the severity of ALI. The left lower lobe was fixed in 4\% paraformaldehyde and embedded in paraffin. Then, the samples were sectioned, stained with hematoxylin and eosin, and examined under a GX53 light microscope (Olympus) at $\times 200$.
The lung injury was scored using a semiquantitative scoring system [14] by the following parameters: 1) alveolar congestion, 2) hemorrhage, 3 ) infiltration or aggregation of neutrophils in airspace or vessel wall, and 4) thickness of alveolar wall/hyaline membrane formation. Each parameter was given one of the following scores: 0, no injury or minimal injury; 1 , mild injury $(<25 \%) ; 2$, moderate injury $(<50 \%) ; 3$, severe injury (up to $75 \%$ ); and 4 , very severe injury (up to $100 \%$ ). The lung injury score was a sum of four parameters (total score: 0-16).

Western blotting. The protein levels of ACSL4 and GPx 4 were assessed by Western blotting. Tissue samples of the right lower lobe of the lung were homogenized in RIPA lysis buffer (Beyotime). Protein concentration was measured using the BCA protein assay kit (Beyotime). The proteins in the cell lysates were separated by SDS-PAGE and transferred to PVDF membranes (Millipore). The primary antibodies were as follows: rabbit anti-ACSL4 (ab227256, Abcam), rabbit anti-GPx4 (ab125066, Abcam), and mouse anti- $\beta$-actin (66009-1-Ig, Proteintech). Subsequently, the membranes were probed with horseradish peroxidase-conjugated secondary antibodies (sc2357, Santa Cruz Biotechnology). The densities of the bands on the membranes were visualized by enhanced chemiluminescence (ChemiDoc XRS+, Bio-Rad), followed by exposure to X-ray film (RX-U, Fujifilm). The results were finally quantified using ImageJ software and expressed as a ratio to $\beta$-actin protein.

RNA extraction and real-time quantitative PCR (RT-qPCR) assay. We used RT-qPCR for evaluation of mRNA expression of ACSL4 and GPx4. The total RNA was extracted from the lung tissues of the right lower lobe using TRIzol reagent (Invitrogen). RT-qPCR was performed with a First-Strand cDNA Synthesis Kit (Fermentas) using an FTC-2000 Real-Time PCR Machine (Funglyn). An equal amount of $\beta$-actin mRNA was used as an internal standard (Proteintech). Primers for ACSL4, GPx4, and $\beta$-actin genes (Table 1) were obtained from Proteintech. The relative expression of each gene was normalized to that of $\beta$-actin using the method of $2^{-\Delta \Delta \mathrm{Ct}}$.

MDA content and SOD activity. MDA and SOD were detected in the lung tissues of the left upper lobe using appropriate kits (Jiancheng Bioengineering Institute) in strict accordance with the manufacturer's protocol.

ELISA. The blood $(4 \mathrm{ml})$ was sampled from the orbit for measuring the levels of TNF $\alpha$ and IL-1 $\beta$. Serum levels of cytokines was assayed by ELISA using Proteintech kits.

Statistical analysis. SPSS 25.0 statistical software (IBM) was used to analyze the experimental data. The data were compared by one-way ANOVA followed by 
Bonferroni correction. The differences were significant at $p<0.05$. The results were presented as $m \pm S E M$.

\section{RESULTS}

Effects of USWD on lung histopathology and lung W/D ratio in rats with LPS-induced ALI. Histological changes in the lung observed in $24 \mathrm{~h}$ after intratracheal instillation of LPS or PBS and their total scores are presented in Figure 1, $a$. In the control group, we observed the normal pulmonary structures. In the ALI group, histopathological changes in the lung tissue included hyperemia and hemorrhage in the alveolar and interstitial space, neutrophil infiltration in airspace and vessel wall, and thickness of the alveolar wall or hyaline membrane formation. In comparison with the control group, the lung injury score in ALI group was strikingly higher $(p<0.001$, Fig. $1, b)$, indicating the successful modeling of ALI in rats. In the USWD group, the histopathological changes in the lungs were significantly attenuated, and the lung injury score was significantly lower than in the ALI group $(p<0.001)$.

The W/D lung weight ratio in the ALI group was significantly higher than in the control group $(p=0.01$; Fig. 1,c); in the USWD group, this parameter showed a decreasing trend $(p=0.544)$. These findings were consistent with the histological results.

Effects of USWD on the expression of ACSL4 and GPx4 and activities of MDA and SOD. The expression of ACSL4 protein and the corresponding mRNA in the ALI group were significantly higher than in the control group $(p<0.001$, Fig. $2, a)$, but USWD could partially block the upregulation of ACSL4 ( $p=0.008, p=0.004$, Fig. $2, b, d$ ). The expression of GPx4 protein and mRNA were significantly decreased in the ALI group compared to the control group $(p<0.001 ;$ Fig. $2, c, e)$. In comparison with ALI group, USWD increased the level of GPx4 protein $(p=0.007$, Fig. $2, c)$. The level of GPx4 mRNA in the USWD group also showed an increasing trend in comparison with ALI group, but the difference was not significant $(p=0.104)$.

TABLE 1. Primer Used for RT-qPCR

\begin{tabular}{l|c}
\hline \multicolumn{1}{c|}{ Gene } & Primer \\
\hline$\beta$-actin & F: ACATCCGTAAAGACCTCTATGCC \\
& R: TACTCCTGCTTGCTGATCCAC \\
ACSL4 & F: CCCTGTCCCGTTCTCATACCG \\
& R: CAAACACCAAAAGGCAACAAGCA \\
GP 44 & F: AATTCGCAGCCAAGGACATCG \\
& R: ATTCGTAAACCACACTCGGCGTA \\
\hline
\end{tabular}

Administration of LPS (ALI group) significantly increased MDA and decreased SOD in the lung tissues in comparison with the control group $(p<0.001$, Fig. 2, $f, g)$. USWD treatment significantly decreased MDA content $(p=0.026)$ and significantly increased SOD activity $(p=0.046)$ in comparison with the ALI group.

Effects of USWD on serum levels of IL-1 $\beta$ and TNFa. In the ALI group, we observed a significant increase in the serum levels of inflammatory cytokines IL-1 $\beta$ ( $p=0.001$; Fig. $3, a)$ and TNF $\alpha(p<0.001$; Fig. 3, $b)$ in comparison with the control group. USWD treatment significantly reduced IL- $1 \beta$ and TNF $\alpha(p=0.036$, $p=0.009$ ), which confirms anti-inflammatory effects of USWD in ALI.

In this study, we explored the mechanisms underlying the anti-inflammatory and anti-oxidant effects of USWD in LPS-induced ALI. We found that USWD reduced the levels of inflammatory cytokines and relieved pathological changes in the lungs of rats with ALI. Moreover, USWD decreased the levels of MDA and ACSL4 and increased the levels of SOD and GPx4. These findings indicated that USWD could effectively alleviate LPS-induced ALI by inhibiting the inflammatory response and oxidative stress. To our knowledge, it is the first study to find the relationship between USWD and oxidative stress on a rat model of LPS-induced ALI.

It is known that ALI leads to severe sequelae and complications, and the incidence of ALI has increased greatly in recent years [6]. Since this disease has important social significance, it has been increasingly investigated by the medical community. Using animal model will be useful to reproduce the pathological state and pathophysiological processes of ALI in humans by stimulating lung tissue damage in rats. LPS, as a major bacterial wall component in gram-negative bacteria, is a well-known and established cause of pneumonia from the community or hospital patients. It has been demonstrated that LPS induces oxidative stress and inflammation both in vivo and in vitro. LPS stimulation is a standard animal model for inducing experimental ALI and ARDS [5].

Inflammation has been reported to play critical roles in ALI [6]. The anti-inflammatory properties of USWD allow using it for various inflammatory diseases [10]. In this experiment, the elevated lung W/D ratio, serum inflammatory cytokines, and neutrophilic infiltration or aggregation in alveoli or vessel walls in the pathological foci in $24 \mathrm{~h}$ after LPS treatment can be considered as a successful inflammatory response. USWD reduced pulmonary edema $(p=0.544)$ and decreased the lung injury score $(p<0.001)$. Although the lung W/D ratio did not decrease significantly, this may be due to the fact that the lung tissues were dried at only $60^{\circ} \mathrm{C}$ (but not at $80^{\circ} \mathrm{C}$ as in other studies [13]). 


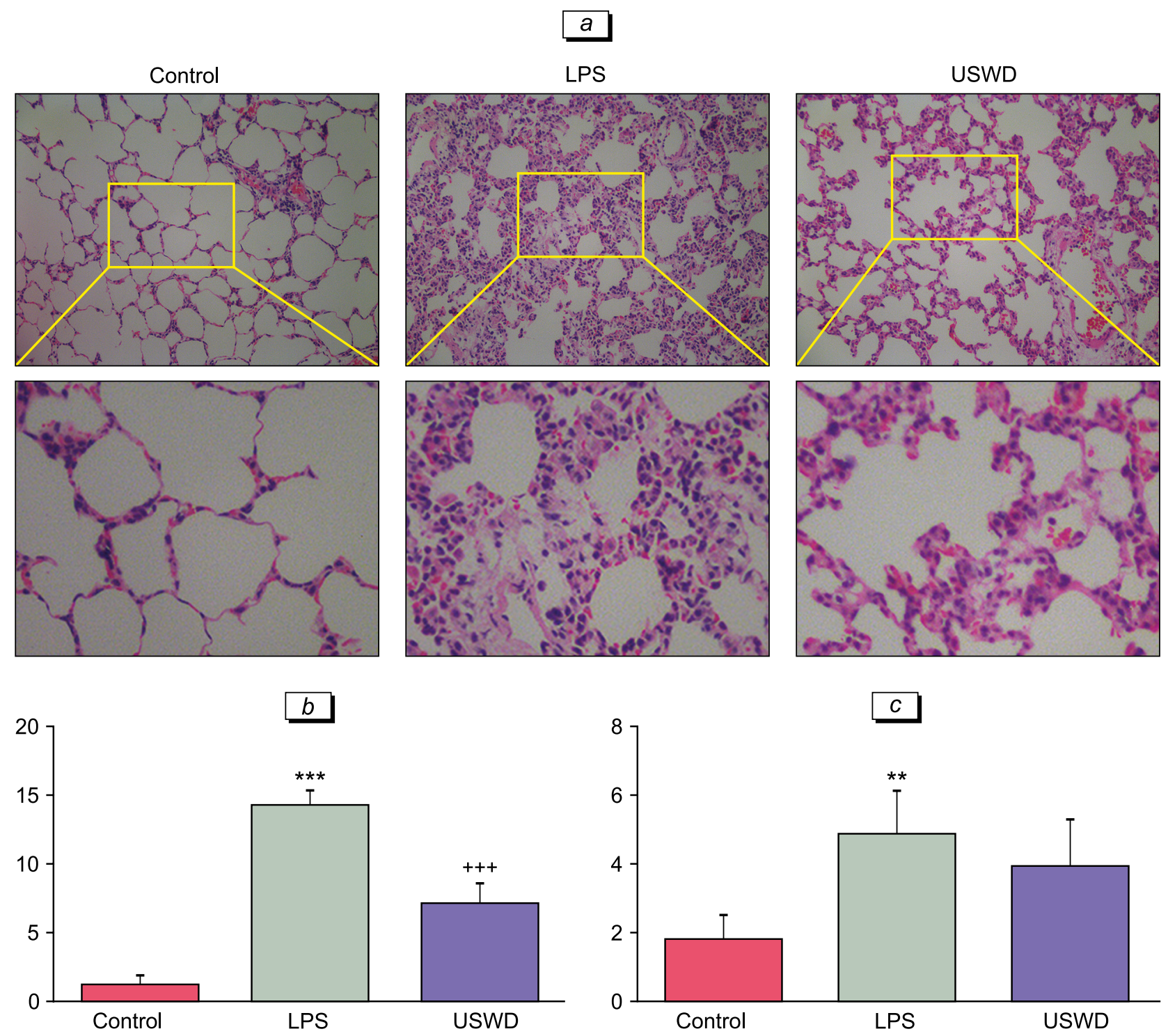

Fig. 1. Effects of USWD on lung damage and lung edema in LPS-induced ALI rats. a) Lung tissue sections stained with hematoxylin and eosin, $\times 200$ (upper row), $\times 600$ (lower row). b) Lung injury score. c) Lung W/D weight ratio. ${ }^{* *} p<0.01,{ }^{* * *} p<0.001$ in comparison with the control group; ${ }^{++} p<0.001$ in comparison with ALI group. Here and in Figs. 2 and 3: results are expressed as $m \pm S E M(n=6)$.

We found that USWD decreased the serum levels of IL-1 $\beta$ and TNF $\alpha$, which attested to the antagonistic role of USWD in local and systemic inflammation. Previous clinical studies have shown that USWD can accelerate resorption of lung exudates, inhibit activity of serum inflammatory cytokines, as well as shorten the course of treatment [15]. A protocol from the China Association of Rehabilitation Medicine Expert Opinion recommended micro-heat USWD could be used to combat COVID-19 in 2020 [15]. Our results further support that USWD alleviates inflammation and has positive effects in ALI.

A number of evidence showed that oxidants and oxidative injury play an important role in the pathogenesis of ALI [3]. Oxidative stress and the resulting inflammation are essential pathological processes in ALI [3]. In vitro and in vivo experiments showed that pathogen-derived and host-derived immune stimuli subsequently contributes to NF- $\kappa \mathrm{B}$ phosphorylation, excessive ROS generation, and secretion of proinflammatory cytokines, which can be the main cause leading to lung injury in animal models [1]. Oxidative stress induces LPO and accumulation of MDA [14]. SOD is an essential anti-oxidant enzyme that converses $\mathrm{O}_{2}^{-}$into $\mathrm{O}_{2}$ and $\mathrm{H}_{2} \mathrm{O}_{2}$ to scavenge ROS. In the present study, MDA content in the lung tissue increased and SOD activity decreased after LPS administration. USWD reduced the intensity of oxidative stress by up-regulating SOD and down-regulating MDA. ACSL4 is involved in the biosynthesis and remodeling 

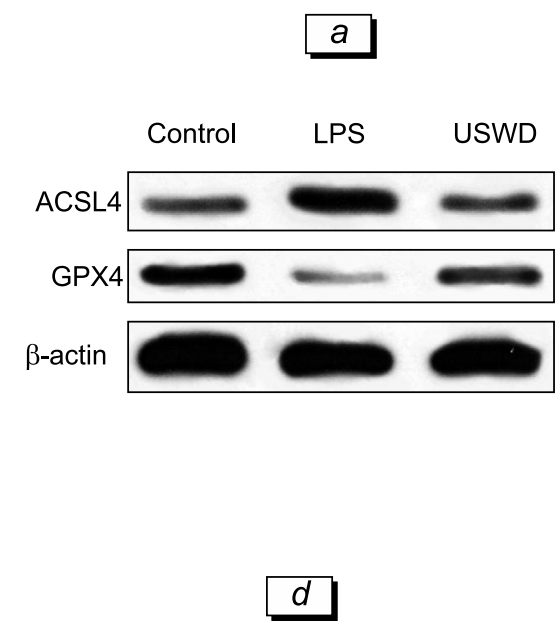

Relative expression of mRNA
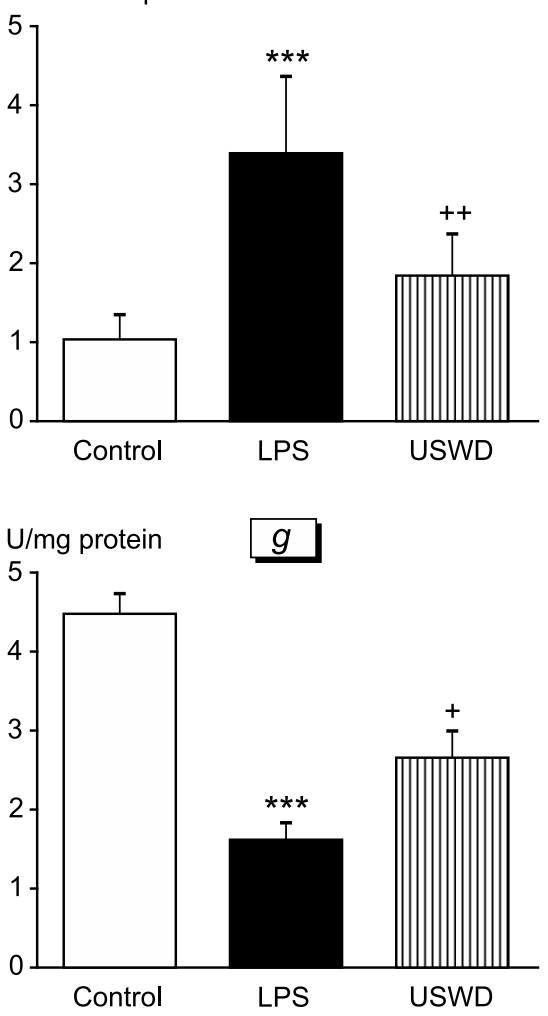

of polyunsaturated fatty acids (PUFAs) and phospholipid hydroperoxides in cell membranes, impairs alveolar barrier by recruiting neutrophils and macrophages and disrupting the integrity of cell plasma membrane, and is indispensable for ferroptotic LPO in ALI [2]. Using glutathione as a cofactor, GPx4 may convert potentially toxic phospholipid hydroperoxides to non-toxic phospholipid alcohols in the cell membrane, which makes it a critical antioxidant enzyme [2]. The elevation of SOD often accompanies the increase of GPx4 [7]. Inhibition of ACSL4 mitigates lung damage by reducing accumulation of LPO substrates in the cell membrane and increasing glutathione and GPx4 levels
Fig. 2. Effects of USWD on the expression of lung ACSL4, GPx4, SOD, and MDA in rats with LPS-induced ALI. a) Expression of ACSL4, GPx4, and $\beta$-actin proteins (Western blotting). $b, c$ ) Relative expression of ACSL4 and GPx4 proteins normalized to $\beta$-actin. $d, e)$ Relative mRNA expression for ACSL4 and GPx4. $f$, g) MDA content and SOD activity in the lung tissue. ${ }^{* * *} p<0.001$ in comparison with the control; ${ }^{+} p<0.05,{ }^{++} p<0.01$ in comparison with ALI group.

[14]. By regulating activation of NF- $\kappa \mathrm{B}$ and oxidation of arachidonic acid, GPx4 activation rebalances glutathione metabolism, decreases ROS level, then intricately lessens inflammatory damages [5]. Low GPx4 and SOD directly leads to an increase in ROS and acute or chronic lung injury [14]. Some studies have also reported that inhibition of ACSL4 or activation of GPx4 can reduce lung damage in ALI rats through preventing hydroperoxide-induced ferroptosis, a form of regulated cell death caused by lethal accumulation of ROS upon iron-dependent lethal LPO in the cell membrane [4]. In the present study, LPS increased ACSL4 and MDA, decreased GPx4 and SOD, and exacerbated 

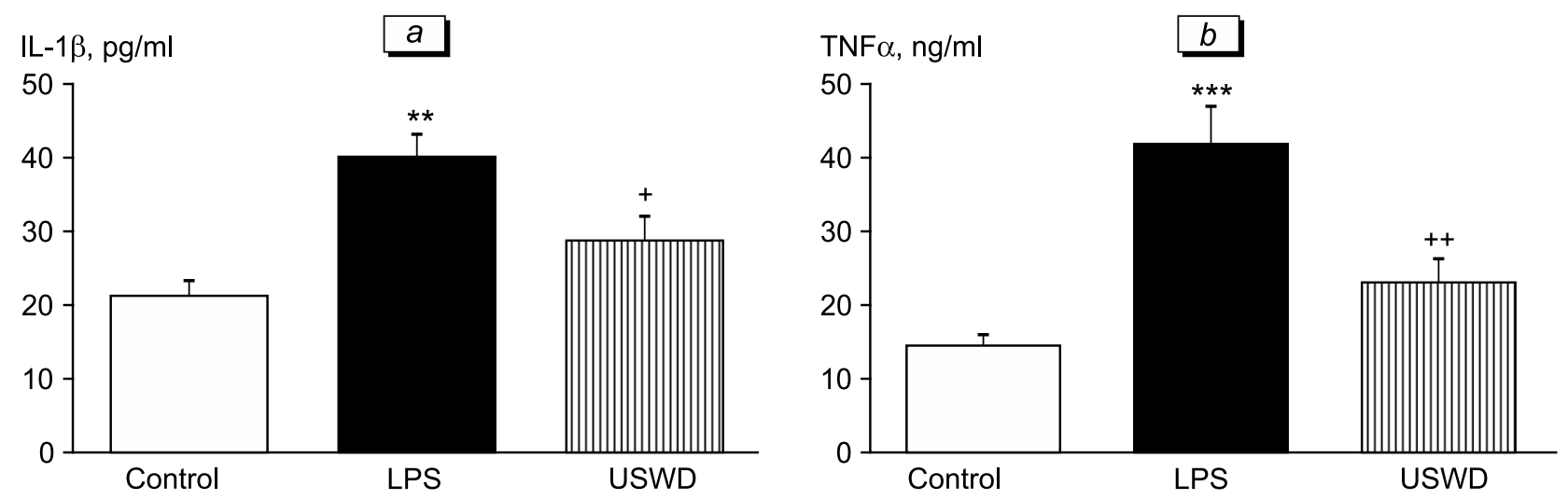

Fig. 3. Effects of USWD on serum production of inflammatory cytokines IL-1 $(a)$ and TNFa $(b)$ in rats with LPS-induced ALI. ${ }^{* *} p<0.01,{ }^{* * *} p<0.001$ in comparison with the control, ${ }^{+} p<0.05,{ }^{++} p<0.01$, in comparison with ALI group.

the inflammatory response in the lung, which is consistent with the findings of previous studies [5].

Previous study has demonstrated that human umbilical cord mesenchymal stem cells combined with USWD therapy could improve motor function in rats with spinal cord injury by inhibiting NF- $\mathrm{KB}$ phosphorylation and attenuating inflammatory microenvironment in spinal cord [12]. We found that USWD not only reduced IL- $1 \beta$ and TNF $\alpha$, but also reversed changes in the content of oxidative stress-related factors after LPS treatment. We further found a dramatic reduction in lung injury score and lung W/D ratio in USWD-treated ALI rats. Therefore, it is reasonable to believe that USWD can attenuate lung injury by inhibiting inflammation and oxidative stress. These evidences support the protective effects of USWD against ALI.

However, limitations still exist in this study. First, the micro-heat USWD might raise body temperature in rats, however we did not measure the temperature during exposure. Second, it should be explored whether anti-oxidant activity is not merely correlated, but is indeed necessary for the positive effects of USWD on ALI.

Clinical studies suggest that USWD may attenuate the lung injury, but the mechanisms are unclear. The biochemical and histopathological results of this study underscore the protective effect of USWD on the lungs through inhibition of inflammation and oxidative stress in LPS-induced ALI model.

USWD can inhibit inflammation and oxidative stress in the lung tissues in LPS-induced ALI rats. USWD can serve as a novel adjuvant therapy to counter inflammation and oxidative stress, and it is a potential therapeutic candidate for the treatment of ALI patients. Our findings have deepened into the mechanisms of USWD against lung injury in LPS-induced ALI rats.

This work was supported by funding from the Natural Science Foundation of Hunan Province (grant No. 2019JJ50536), Foundation of Health and Fami- ly Planning Commission of Hunan Province (grant No. C20180238), Hengyang Science and Technology Development Foundation (grant No. HKF201947209), and Sub-specialty key project of The First Affiliated Hospital of University of South China (grant No. 2017ZD002).

The authors have no relevant financial or non-financial interests to disclose. The authors have no conflicts of interest to declare that are relevant to the content of this article.

\section{REFERENCES}

1. Dong Z, Yuan Y. Accelerated inflammation and oxidative stress induced by LPS in acute lung injury: Inhibition by ST1926. Int. J. Mol. Med. 2018;41(6):3405-3421. doi: 10.3892/ ijmm.2018.3574

2. Imai H, Matsuoka M, Kumagai T, Sakamoto T, Koumura T. Lipid Peroxidation-Dependent Cell Death Regulated by GPx4 and Ferroptosis. Curr. Top Microbiol. Immunol. 2017;403:143170. doi: $10.1007 / 82 \quad 2016508$

3. Kellner M, Noonepalle S, Lu Q, Srivastava A, Zemskov E, Black S.M. ROS Signaling in the Pathogenesis of Acute Lung Injury (ALI) and Acute Respiratory Distress Syndrome (ARDS). Adv. Exp. Med. Biol. 2017;967:105-137. doi: 10.1007/978-3-319-63245-2_8

4. Latunde-Dada GO. Ferroptosis: Role of lipid peroxidation, iron and ferritinophagy. Biochim. Biophys. Acta Gen. Subj. 2017;1861(8):1893-1900. doi: 10.1016/j.bbagen.2017.05.019

5. Lei J, Wei Y, Song P, Li Y, Zhang T, Feng Q, Xu G. Cordycepin inhibits LPS-induced acute lung injury by inhibiting inflammation and oxidative stress. Eur. J. Pharmacol. 2018;818:110-114. doi: 10.1016/j.ejphar.2017.10.029

6. Li T, Wu YN, Wang H, Ma JY, Zhai SS, Duan J. Dapk1 improves inflammation, oxidative stress and autophagy in LPSinduced acute lung injury via $\mathrm{p} 38 \mathrm{MAPK} / \mathrm{NF}-\kappa \mathrm{B}$ signaling pathway. Mol. Immunol. 2020;120:13-22. doi: 10.1016/j.molimm.2020.01.014

7. Li W, Li W, Leng Y, Xiong Y, Xia Z. Ferroptosis Is Involved in Diabetes Myocardial Ischemia/Reperfusion Injury Through Endoplasmic Reticulum Stress. DNA Cell. Biol. 2020;39(2):210225. doi: 10.1089/dna.2019.5097 
8. Li Y, Cao Y, Xiao J, Shang J, Tan Q, Ping F, Huang W, Wu F, Zhang $\mathrm{H}$, Zhang $\mathrm{X}$. Inhibitor of apoptosis-stimulating protein of p53 inhibits ferroptosis and alleviates intestinal ischemia/reperfusion-induced acute lung injury. Cell. Death Differ. 2020;27(9):2635-2650. doi: 10.1038/s41418-0200528-x

9. Mowery NT, Terzian WTH, Nelson AC. Acute lung injury. Curr. Probl. Surg. 2020;57(5):100777. doi: 10.1016/j.cpsurg.2020.100777

10. Shields N, Gormley J, O'Hare N. Short-wave diathermy: current clinical and safety practices. Physiother. Res. Int. 2002;7(4):191-202. doi: 10.1002/pri.259

11. Wang N, Feng Z, Zhao W, Zhang Z, Zhang L. Ultrashortwave radiation promotes the recovery of spinal cord injury by inhibiting inflammation via suppression of the MK2/TNF- $\alpha$ pathway. Int. J. Mol. Med. 2018;42(4):1909-1916. doi: 10.3892/ ijmm.2018.3786
12. Wang S, Jia Y, Cao X, Feng S, Na L, Dong H, Gao J, Zhang L. HUCMSCs transplantation combined with ultrashort wave therapy attenuates neuroinflammation in spinal cord injury through NUR77/NF-кB pathway. Life Sci. 2021;267:118958. doi: $10.1016 /$ j.lfs.2020.118958

13. Wang X, Deng R, Dong J, Huang L, Li J, Zhang B. Eriodictyol ameliorates lipopolysaccharide-induced acute lung injury by suppressing the inflammatory COX-2/NLRP3/NF- $\mathrm{KB}$ pathway in mice. J. Biochem. Mol. Toxicol. 2020;34(3):e22434. doi: $10.1002 / j b t .22434$

14. Xu Y, Li X, Cheng Y, Yang M, Wang R. Inhibition of ACSL4 attenuates ferroptotic damage after pulmonary ischemia-reperfusion. FASEB J. 2020;34(12):16262-16275. doi: 10.1096/ fj.202001758R

15. Yu HP, Jones AY, Dean E, Liisa Laakso E. Ultra-shortwave diathermy - a new purported treatment for management of patients with COVID-19. Physiother. Theory Pract. 2020;36(5):559-563. doi: 10.1080/09593985.2020.1757264 